\title{
Representation of Long-Time Creep in a Pure-Gum Rubber Vulcanizate
}

\author{
Lawrence A. Wood* \\ National Bureau of Standards, Washington, D.C. 20234
}

January 30, 1980

\begin{abstract}
Creep may be expressed as $\Delta E / E_{1}=A \log t+B(t-1)$ where $\Delta E$ is the increase of elongation above $E_{1}$ the one-minute value, during the time $t$. The initial slope of a plot of $\Delta E / E_{1}$ against $\log t$ is $A$, a measure of physical creep. The limit of the final slope of a plot of $\Delta E / E_{1}$ against $(t-1)$ is $B$, a measure of chemical creep. The validity of the equation is determined by the linearity of a plot of $\left(\Delta E / E_{1}-A \log t\right)$ against $(t-1)$ after $A$ has been determined by the first plot. $B$ is obtained as the slope. The equation is almost always valid and simultaneous equations can be used to determine $A$ and $B$ from only three observations, if desired. $A$, usually between 1 and 10 per cent/unit $\log t$, is strongly dependent on cross-linking and nearly independent of temperature. $B$, ranging from $0.1 \times 10^{-5}$ to $10,000 \times 10^{-5}$ per cent/min is nearly independent of cross-linking and strongly dependent on temperature and specimen thickness. With an activation energy of $84-125 \mathrm{~kJ}(\mathrm{~mol})^{-1}\left(20-30 \mathrm{kcal}(\mathrm{mol})^{-1}\right)$ it probably reflects oxidative degradation of the network, often initiated by ozone. The appearance time at which the creep is first observed to exceed $A \log t$ can be taken as equal to $B^{-1}$. At high temperatures $B$ is drastically increased with a corresponding strong reduction in appearance time. Creep in excess of that given by the equation is sometimes observed during a period immediately before rupture.
\end{abstract}

Key words: Rubber: creep of; long-time creep of; mechanical properties of; relaxation of; time effects in.

\section{Introduction}

The deformation of rubber specimens subjected to a constant uniaxial stress is always found to increase with time. At first the increase of deformation is usually nearly linear with the logarithm of the time and at long times it becomes nearly linear with the time itself.

If the elongation in simple uniaxial extension is denoted as $E$ (others have often used $e$ or $\varepsilon$ ) and its value at unit time is $E_{1}$, the following equation is found to be widely applicable.

$$
\left(E-E_{1}\right) / E_{1}=\Delta E / E_{1}=A \log \mathrm{t}+B(\mathrm{t}-1)
$$

$A$ and $B$ are constants independent of time. A few unusual instances in which $B$ increases or decreases with time are discussed in a later section, but the equation is found to be valid for the great majority of rubber vulcanizates investigated.

It will be noted that the two terms in the equation are both zero at unit time (where $\log t=0$ ). The time unit chosen in the present study is one minute. Lyons [1] ${ }^{1}$ has

\footnotetext{
- Center for Materials Science, National Measurement Laboratory.

${ }^{1}$ Figures in brackets indicate literature references at the end of this paper.
}

pointed out that the equation is of very general applicability. He gives a comprehensive historical summary of its application to many amorphous and polycrystalline solids of widely different molecular structures. With the exception of the work of Gent [2] and Derham [3-5], its application to rubber is fairly limited, largely because most creep studies have not been continued to long times. The equation was used by the present author and his colleagues [6] in a study of the influence of humidity and atmospheric oxygen on creep. Reference should be made to that publication for an extensive summary of the limited amount of earlier work on long-time creep of rubber.

The present paper is devoted to methods of representing data on long-time creep, including a study of the equation. Procedures are developed for evaluating the constants from a minimum amount of experimental data.

\section{Equation for representing creep as a function of time}

Equation (1.1) shows as ordinate the fractional creep $\Delta E / E_{1}$ (rather than $\Delta \mathrm{E}$ itself), since $\Delta E / E_{1}$ varies relatively little with $E_{1}$ over a considerable range of elongations [2]. 
This facilitates the comparison of different sets of data when $E_{1}$ is not the same because different constant stresses have been used. For a detailed study of eq (1.1) it is convenient to make a double-abscissa plot like that shown in figures 1 and 2, each showing two curves for $\Delta E / E_{1}$-one with the lower abscissa $\log t$ (lower curve) and one with the upper abscissa $(t-1)$ (upper curve) [6]. Figure 1 shows the effect of increasing values of $B$, when $A$ is held constant, while in figure $2 A$ is increased with $B$ constant. The maximum time shown in each figure is 100,000 min. (about 70 days), where the two abscissa scales are made to coincide.

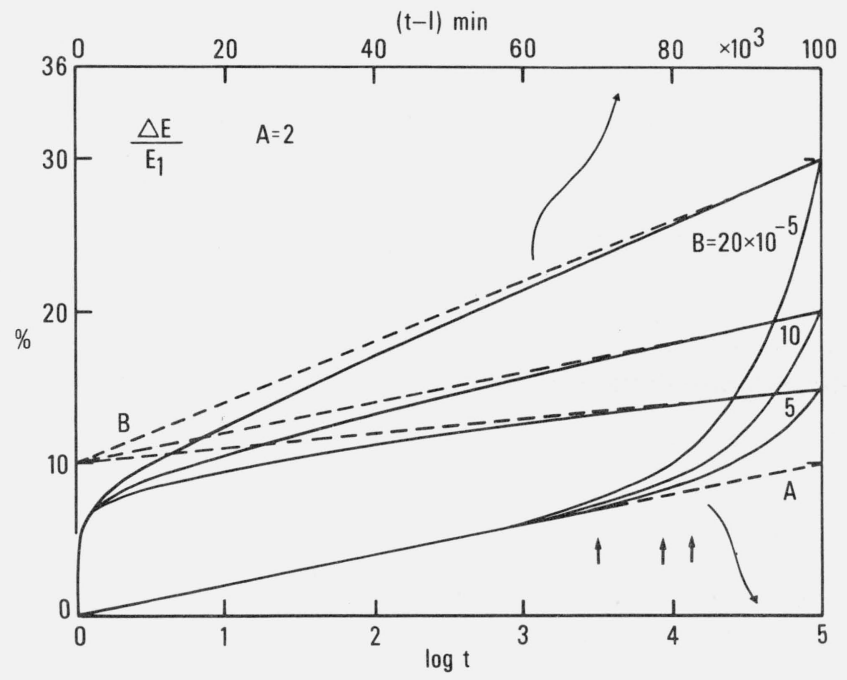

FIGURE 1. Fractional creep as a function of log $\mathrm{t}$ or of $(\mathrm{t}-1)$ $A=2$ percent/unit $\log t$ $B=5,10$, or $20 \times 10^{-5}$ percent $/ \mathrm{min}$.

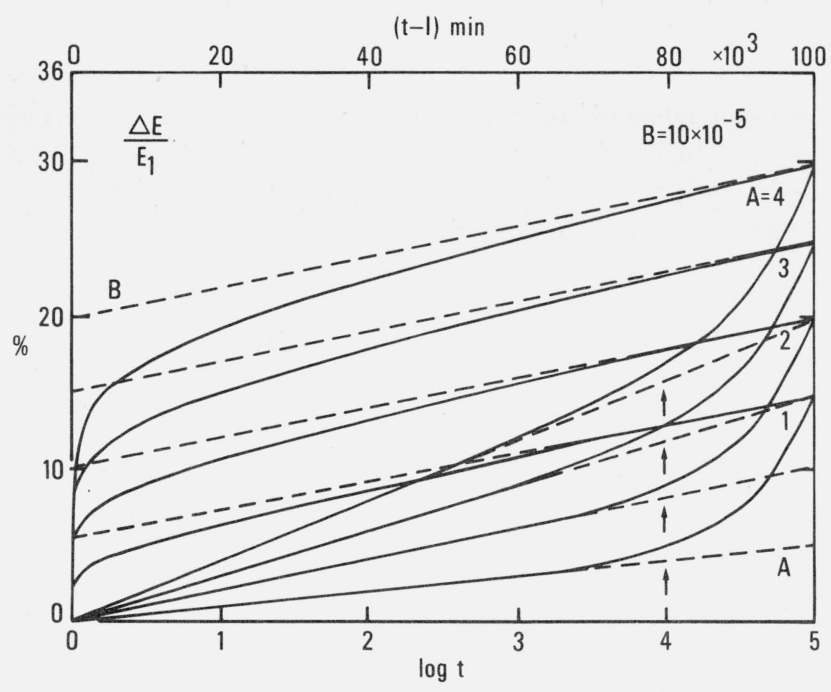

FIGURE 2. Fractional creep as a function of log $\mathrm{t}$ or of $(\mathrm{t}-1)$ $A=1,2,3$, or 4 percent/unit $\log t$ $B=10 \times 10^{-5}$ percent $/ \mathrm{min}$.
The slopes of the two curves are found by differentiating eq(1.1)

$$
\begin{aligned}
& \frac{\partial}{\partial \log t} \frac{\Delta E}{E_{1}}=A+2.303 B t \\
& \frac{\partial}{\partial t} \frac{\Delta E}{E_{1}}=0.4343 A / t+B
\end{aligned}
$$

It is obvious that the values given by eq (2.1) approach $A$ as $t$ is reduced and those given by eq (2.2) approach $B$ as $t$ is increased. This behavior can be noted in figures 1 and 2 , where dashed lines are drawn having the slopes $A$ and $B$. Our previous paper [6] erroneously omitted the numerical constant in giving eq (2.1).

The form of eq (1.1) may be readily explained if it is assumed that there are two different processes giving rise to creep. The first-predominating at short times-is characterized by values of the constant $A$. It is found to be fully recoverable [7] and has been called "physical creep" or "primary creep." The second process-predominating at long times-is characterized by values of the constant B. It is found to be only partially recoverable [2] in many instances and has been called "chemical creep" or "secondary creep." Both processes are assumed to be effective throughout the whole range of time with the range of predominance of physical creep being determined by the magnitude of the constant $B$. There is usually an extensive intermediate region where neither process predominates.

Typical experimental data obtained in our previous study [6] are presented in figure 3. The experimental points are well-represented by the two curves, which are plots of eq.

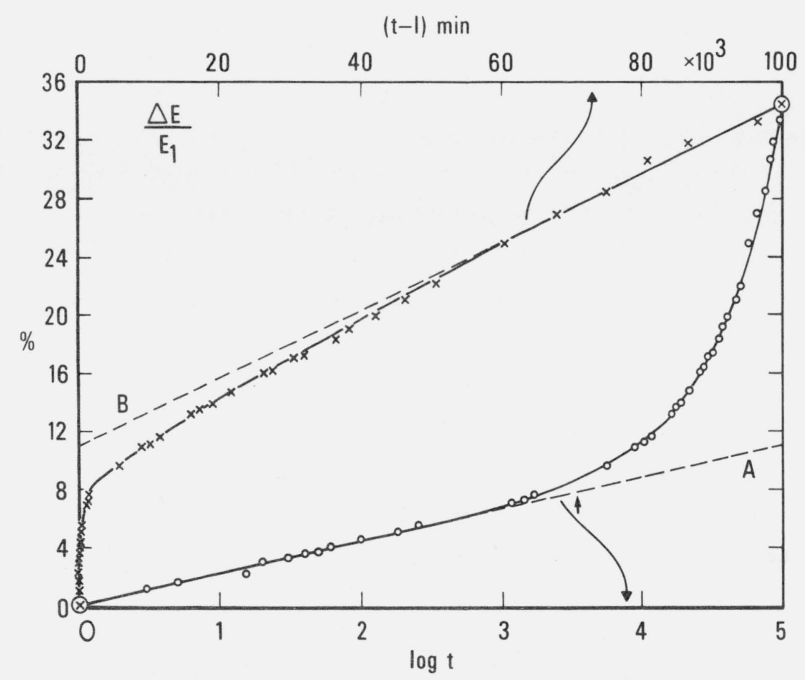

FIGURE 3. Typical fractional creep as a function of log $\mathrm{t}$ or of $(\mathrm{t}-1)$

$$
\begin{aligned}
& A=2.239 \text { percent/unit } \log t \\
& B=23.59 \times 10^{-5} \text { percent } / \mathrm{min} . \\
& \text { Data of Wood, Bullman, and Roth [6] }
\end{aligned}
$$


(1.1) taking $A$ as 2.239 percent/(unit $\log t)$ and $B$ as $23.59 \times$ $10^{-5}$ percent $/ \mathrm{min}$. Similar agreement with the equation has been found for almost all the data on long time creep of rubber found in the literature. Exceptions will be described in a later section.

Double-abscissa graphs like those in figures 1,2 , and 3 show the physical creep at $10^{5} \mathrm{~min}$. as the ordinate of straight line $\mathrm{A}$ at $10^{5} \mathrm{~min}$. The intercept of line $B$ (when $t=$ 1 and $\log t=0$ ) has the same value. The chemical creep at $10^{5} \mathrm{~min}$ is then the difference between the total creep and this intercept. One can readily note by examining the graphs the relative sizes of physical and chemical creep at $10^{5} \mathrm{~min}$.

\section{Graphical evaluation of $A$}

The constant $A$ in eq. (1.1) can be determined by measuring the slope of the graph of $\Delta E / E_{1}$ against $\log t$ over its region of initial linearity. In much of our work $[8,9]$ this has involved measurements over the range from 1 to more than 1,000 minutes. Other work $[10,11]$ has indicated that the lower limit might be extended to times of the order of seconds or even milliseconds if desired. Indentation-time measurements [12-14] are also useful in this connection. The upper limit should be the point at which it is apparent that the values of $\Delta E / E_{1}$ are above those given by the line representing the earlier values. A quantitative evaluation of this limit in terms of the constant $B$ is given in section 6 of the present paper.

The values of $A$ in studies of the creep of conventional rubber vulcanizates have usually ranged between 1 and 10 percent/unit $\log t$. In general it is highly sensitive to the amount of cross-linking, and much lower values are found for some highly cross-linked systems $[9,14]$. It is also highly dependent on the structure and composition of the rubber.

\section{Graphical evaluation of B}

The constant B in eq (1.1) can be determined by estimating the limiting slope of the graph of $\Delta E / E_{1}$ against $(t-1)$ at the longest times. It is often quite difficult to estimate accurately this limiting slope, since the value is changing slowly. Consequently the procedure proposed by Gent [2] and illustrated in figure 4 is recommended as a preferable alternative.

In this procedure the constant $A$ is first evaluated, as discussed in the previous section. It can be readily seen from eq (1.1) that by plotting $\left(\Delta E / E_{1}-A \log t\right)$ against $(t-1)$, as in figure 4, one should obtain a straight line with the slope B and an intercept of zero. Furthermore if the results are not represented by eq (1.1) a deviation from the line will occur.

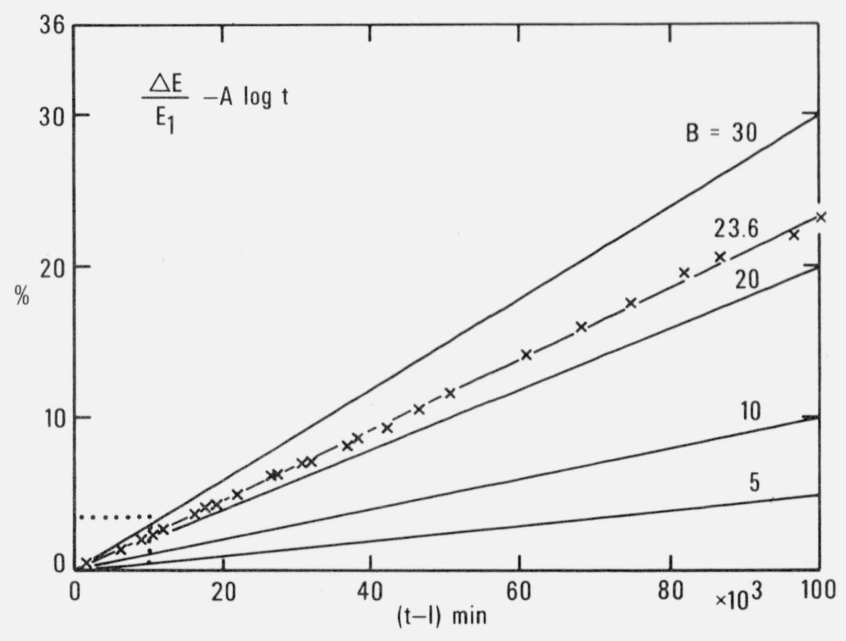

FIGURE 4. Chemical creep

$\left(\Delta E / E_{1}-A \log t\right)$ as a function of $(t-1)$

$A=2.239$ percent/unit $\log t$

Data of Wood, Bullman, and Roth [6]

Figure 4, shows that the experimental data presented in figure 3 do indeed conform to the equation and that the slope corresponds to the constant $B$ and the intercept is zero. Lines corresponding to other values of $B$ are also shown.

Values of $B$ obtained in various studies of rubber vulcanizates cover a very wide range from as low as $0.1 \times 10^{-5}$ percent/min to as high as $10,000 \times 10^{-5} /$ percent $\min$.

\section{Analytical evaluation of $A$ and $B$}

As an alternative to the graphical determination of the constants $A$ and $B$ described in the preceding sections one may determine them both at the same time by a simple analytical procedure if he is convinced that the data conform to eq (1.1), and $E_{1}$ has been determined. The analytical procedure merely involves obtaining the two constants by solving the two simultaneous equations obtained by inserting two observed values of $\Delta E / E_{1}$ into eq (1.1). In our work it was found desirable of course to utilize $\Delta E / E_{1}$ at the longest time (usually about $10^{5} \mathrm{~min}$.) as one of the values. The choice of the time (usually about $10^{3} \mathrm{~min}$.) selected for the other value made very little difference in the values found for $A$ and $B$. The analytical method can not be applied when the data do not conform to eq. (1.1), as in the pre-rupture studies described in section 8 or in other cases where the graphical method shows that B is increasing or decreasing with time.

The analytical method is regarded as more accurate and precise than the graphical method in most cases. The constants given for the data of figure 3 were determined by the analytical method, and the graphs are representations of eq. (1.1) utilizing the constants. All the experimental points 
show very good agreement with the curves although only two of them were used in determining $A$ and $B$.

The minimum number of experimental observations to determine the creep curves is thus seen to be two, in addition of course to $E_{1}$ the one-minute elongation. In the extreme then, for example, it is sufficient to make measurements at 1, 1,000, and 100,000 min.

If it is desired to complete the evaluation of the creep in the shortest possible time rather than with the minimum number of observations, consideration should be given to the use of the plot of $(\Delta E / E-A \log t)$ against $(t-1)$, as in figure 4. The slope of this line, which gives the constant $B$, can be determined from the early observations. For example, it is clear from this graph that this slope could have been obtained without using any observations beyond 10,000 min. The precision of the observations and the magnitude of $\mathbf{B}$ will determine the minimum time required to evaluate this slope. This linear extrapolation of data of the type plotted in figure 4 should be extremely valuable in dealing with the very common problem of predicting creep over a period of years utilizing data extending only over much shorter periods. It should be emphasized that no change in predicted behavior is indicated as the observations are extended to longer and longer times.

\section{Appearance time and linearity limit}

It is clear from figures 1,2 , and 3 that there is a region (short times) where $\Delta E / E_{1}$ appears to be linear with $\log t$. It is of interest to examine the upper boundary of this region where it first becomes apparent that $\Delta E / E_{1}$ is greater than $A \log t$. The time at which one can be sure that this is true will depend on the sensitivity of the method of measurement. This boundary has been considered by several previous authors, but often the concept has been rather vague and has lacked a rigorous definition. The term "appearance time" used by Metherell [15] for the boundary might give a misleading implication that a new process begins at this point. However, we shall continue to use it since the alternatives "induction time" or "incubation time" used by Aben $[16,17]$ are even more open to the same objection. Neither of these authors made quantitative definition of the term.

In order to establish an arbitrary quantitative standard for defining appearance time let us assume that from experimental observations one can very readily determine the time $t_{a}$ at which the observed value of $\Delta E / E_{1}$ is one unit(i.e. $1 \%)$ greater than $A \log t_{a}$. Thus from eq (1.1)

$$
\Delta E / E_{1}-A \log t_{a}=B\left(t_{a}-1\right)=1
$$

This relation between $B$ and $\left(t_{a}-1\right)$ is represented by the upper straight line of figure 5 .

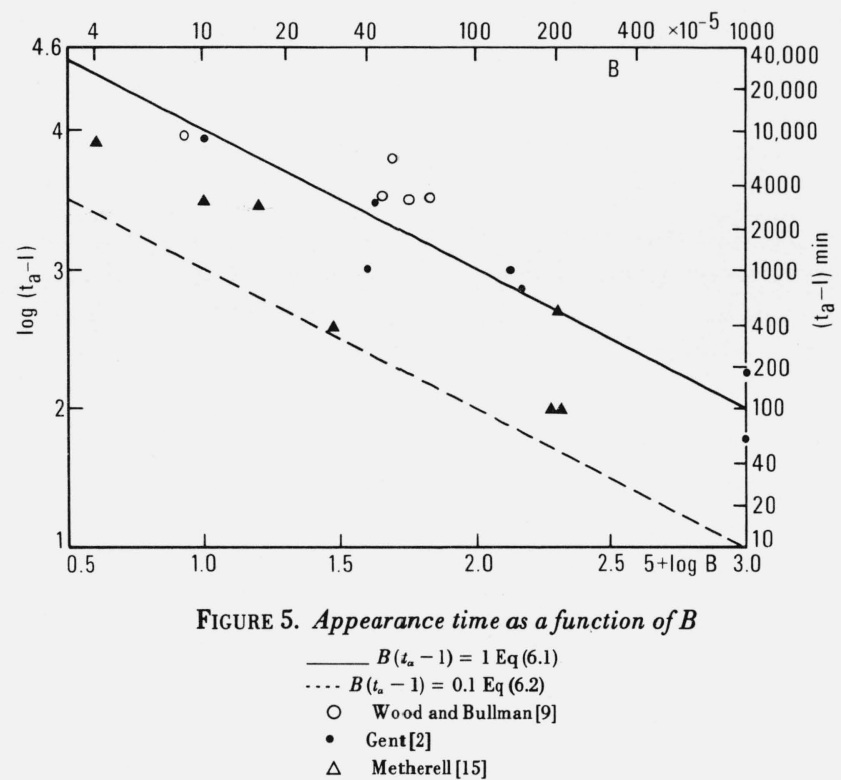

The arrows in figures 1, 2 and 3 show the location of the appearance time, as calculated from $B$ by eq (6.1.) In these figures a reasonable estimate of its location could have been made by a direct examination of the curves. In contrast, plots of chemical creep like figure 4 show no indication of an appearance time.

Under the proper experimental conditions it seems that the appearance time might be determined with greater accuracy than the one unit considered in the definition just given. If under favorable conditions it could be determined to 0.1 unit an alternative definition would be

$$
B\left(t_{a}-1\right)=0.1
$$

This equation is represented by the lower straight line in figure 5 . The values of $\log \left(t_{\alpha}-1\right)$ in this equation are of course exactly one unit less than those given by equation (6.1)

The discussion of $t_{a}$ as the time at which the effects of the $B^{-}$term first became evident does not imply a discontinuity of slope of figures 1, 2 and 3 or that a new type of behavior begins at these times. The $B$-process has been in existence from the beginning of the experiment, but previously the $B$-term has been too small to be observed.

This behavior was not understood by the present author in 1972, [9] when he proposed to explain a marked increase of slope of creep curves in rubber cross-linked by dicumyl peroxide as being due to the initiation of crystallization after about 2000 min. of creep. Derham [18] later suggested that the increased creep was due to humidity, but Metherell [15] in more extensive work related it to ozone in the atmosphere. It now appears that the ozone gave rise to a high value of $B$ throughout the experiment, and that $2000 \mathrm{~min}$ was 
merely the appearance time under the conditions of the experiment. The appearance time did not vary significantly with cross-linking.

The data of Wood and Bullman [9] (some of it not reported in the original publication) extending to a time of 20,000 min. have now been plotted to show chemical creep $\left(\Delta E / E_{1}\right.$ $-A \log t)$. Straight lines like those in figure 4 were obtained with constant slopes showing values of $B$ lying largely in the range $45-65 \times 10^{-5}$ percent $/ \mathrm{min}$. The values of $B$ depended very little on the amount of cross-linking as would be expected from the observation that the appearance times did not depend on the cross-linking. The value of $A$, on the other hand, was chiefly determined by the amount of crosslinking, becoming negligible at the highest amounts.

Equation (6.1) plotted in figure 5 shows that the values of $B$ just given correspond to an appearance time of the general magnitude of $2000 \mathrm{~min}$. (where $\log t=3.3$ ). The detailed experimental values are plotted in figure 5 together with all similar data reported for corresponding values of $B$ and $\log t_{a}$ in the papers by Gent [2] and Metherell [15]. One concludes from figure 5 that the criterion defining $t_{a}$ in eq. (6.1) is not far different from that actually employed in the respective publications. Some of Metherell's data were closer to the values obtained in eq. (6.2). In the creep studies of Meier, Rudd, and Weir [19] it was found that at times between 1000 and 10,000 min the plot of creep against log time began to deviate from linearity because chemical creep was becoming appreciable.

The criterion for defining appearance time used to derive eq (6.1) or (6.2) is not the only one that might have been used. Indeed in our previous work [6] appearance time was defined in terms of a 10 percent difference in slopes. The resulting equation expressed the appearance time as a function of the quantity $B / A$ rather than $B$ alone as found here. In the work of Wood and Bullman [9] just mentioned, a very large variation in the degree of cross-linking which led to a very large variation in the constant $A$ (even to the point where $A$ was zero within experimental error) produced no systematic variation in appearance time. This fact is considered as additional strong evidence that the criterion used in the present paper was more satisfactory than that used earlier.

At sufficiently long times there is a region in which $\Delta E / E_{1}$ is found to be approximately linear with time. The lower boundary of the region may be called $t_{b}$ the linearity limit. The literature contains very few quantitative evaluations of $t_{b}$. Conceptually it is distinctly different from $t_{a}$, the appearance time, although some writers have made little or no distinction between them. Establishing a quantitative definition of $t_{b}$ is more difficult and arbitrary than the corresponding establishment of the definition of $t_{a}$.

Straight line $A$ in figures 1,2 , and 3 always passes through the origin and its location is therefore determined uniquely by specifying its slope. Straight line $B$ (which $\Delta E / E_{1}$ approaches at long times), on the other hand, is not constrained to pass through any specified point. Only its slope is specified, and the line itself is steadily displaced upwards the higher the value of the observed time. In figures 1,2 , and 3 the line has been arbitrarily forced to pass through the value of $\Delta E / E_{1}$ at $\mathrm{t}=100,000 \mathrm{~min}$., since observations rarely extend to any longer times. This selection of a particular value introduces an arbitrary element into the definition and we shall not attempt to propose a quantitative definition of the linearity limit in this paper. Normally the linearity limit is considerably larger than the appearance time.

\section{Comparison with stress relaxation}

The present paper has been concerned with the increase of extension under a fixed load (creep). Gent $[2,7]$ some years ago pointed out the close relationship between creep and the decrease of stress at a fixed extension (stress relaxation). For example either $\log \left(F / F_{1}\right)$ or the ratio $\Delta F / F_{1}=$ $\left(F-F_{1}\right) / F_{1}$ (where $F$ is the stress at a time $t$ and $F_{1}$ the stress at unit time) is often found to decrease proportionally to log $t$ at short times and to be decreasing linearly with $t$ at long times. The time beyond which the ratio no longer appears to be proportional to $\log t$ is approximately the same as the corresponding appearance time in the creep studies. The available data appear to be insufficient to determine whether the linearity limit for stress relaxation is the same as that for creep.

The data on stress relaxation found in the literature are much more extensive than the data on creep. The results of studies of appearance time for stress relaxation and its change with temperature are especially valuable for comparison with the corresponding creep studies. Many years ago Tobolsky and his collaborators [20], [21] showed that, following viscoelastic effects, the most important reaction in many cases is oxidative degradation of the network chains with an activation energy near $125 \mathrm{~kJ}(\mathrm{~mol})^{-1}(30 \mathrm{kcal}$ $(\mathrm{mol})^{-1}$. The later stress relaxation studies of Thirion and Chasset [22-24], Aben [16, 17] and Tamura and Murakami $[25,26]$ are quite parallel to the present work on creep. Further discussion of stress relaxation data appears in Section 9 of the present paper dealing with the effects of temperature.

\section{Significance of B}

The constant $B$ is a measure of what is sometimes called "chemical creep" and sometimes "secondary creep". It includes creep arising from degradation of the network and any other reactions proportional to time. 
Where the constant $A$ is very much governed by the degree of cross-linking, the constant $B$ (and consequently the appearance time $t_{a}$ ) is almost independent of the degree of cross-linking. This is evident in the studies of natural rubber cross-linked by dicumyl peroxide already mentioned [9]. The logarithm of the appearance of time varies only from 3.5 to 3.8 as the cross-linking was raised from low values to about $150 \mathrm{~mol} / \mathrm{m}^{3}$. The constant $B$ at the same time remained in the range 45 to $65 \times 10^{-5}$ percent $/ \mathrm{min}$ (as mentioned in Section 6) and did not vary systematically with the degree of cross-linking. Therefore one may conclude that the degradation reflected by $B$ occurs in the main chains and not in the cross-links. This is in accordance with Tobolsky's conclusions regarding natural rubber [20], [21].

Where the constant $A$ is influenced relatively little by temperature or ambient conditions such as the presence of ozone, oxygen, or humidity, the constant $B$ is extremely sensitive to them. [6] $B$ is therefore a very useful parameter for relative comparisons of different rubbers and different conditions. Unfortunately many of the observations of creep in the literature do not extend to times long enough to evaluate it accurately by the methods given in the preceding sections. Often the observations were discontinued soon after a vaguely-defined "appearance time" was reached. It is not feasible to draw conclusions regarding $B$ from experimental data until the effect of physical creep, represented by $A$, has been eliminated. In future work it is strongly recommended that observations extend to times long enough to permit the accurate evaluation of $B$ by the methods given here.

Gent has presented a most comprehensive and significant paper [2] opening up this field. He evaluated the coefficient $B$ for a number of different rubber vulcanizates and interpreted the results in terms of molecular considerations. In this manner he was able to draw conclusions regarding oxidative scission, breaking and stabilization of cross-links, effects of extraction, effects of long time cures, and many other operations. This is a very fruitful field for further investigation.

In Gent's work two instances were reported in which the coefficient $B$ varied with time so that eq (1.1) was not applicable. In one case $B$ showed continuous increase ascribed to auto-catalysis already noted in stress relaxation measurements [27] and in the other it showed a decrease for a specimen in vacuo before becoming approximately constant. The latter behavior was interpreted in terms of the abnormal situation of a cross-link structure changing with time. These cases illustrate the importance of plotting secondary creep against $(t-1)$ to determine whether eq. (1.1) is applicable. Gent found linearity with $(t-1)$ for the great majority of the vulcanizates he studied.

Although the evaluation of $B$ is useful as a means of comparing different rubbers in relation to each other it can not be regarded as a genuine characteristic property of a rub- ber since it depends on the thickness of the specimen. In fact a plot of $B$ against the reciprocal of the thickness was found in at least one instance [15] to have an intercept of zero. This means that $B$ would vanish for a specimen of infinite thickness. In this case the value of $B$ was largely dependent on the amount of ozone present, and time was required for it to diffuse inward from the surface of the specimen. The creep was thus diffusion-controlled. At room temperature with thick specimens in the absence of appreciable amounts of ozone the values of $B$ are normally quite small. The thickness of the specimens in our previous work on long-time creep [6] was only $0.5 \mathrm{~mm}$. so that the creep would not be diffusion-controlled.

One of the lowest values of $B$ reported in the literature is 1 percent yer year, equivalent to about $0.2 \times 10^{-5}$ percent per min., as given by Derham [3] for an efficiently vulcanized natural rubber $1 \mathrm{~mm}$ thick at $15^{\circ} \mathrm{C}$.

Meier, Rudd, and Weir [19] made extensive creep studies for periods up to about 2 years. Unfortunately they did not report values of $\Delta E / E$, at short times, from which the constant $A$ could be determined. However, from the values reported at long times upper limits could be set for values of $B$. These maximum values were usually in the range from $0.1 \times 10^{-5}$ to $2 \times 10^{-5}$ percent per min. for the formulations showing the lowest long-time creep. They were thus in the general range of 1 percent per year given by Derham. [3]

For values of $B$ above $10,000 \times 10^{-5}$ percent per min. the appearance time $t_{a}$ calculated from eq (6.1) is so short (less than $11 \mathrm{~min}$.) that experimental observations usually have not yielded a reliable value of $t_{a}$.

\section{Effects of temperature}

The constant $A$ is nearly independent of temperature. Indentation studies [14], [29] extending to only $10 \mathrm{~min}$ showed no change of creep rate as the temperature was raised from about 20 to $100^{\circ} \mathrm{C}$. Gent [2] showed values for a stretched natural rubber vulcanizate at $60{ }^{\circ} \mathrm{C}$ to be slightly smaller than at $23^{\circ} \mathrm{C}$ for times up to $1,000 \mathrm{~min}$. Somewhat similar behavior was noted for natural rubber by Derham [5] and for the Butyl rubber (GR-I) studied by Martin, Roth and Stiehler [8] at times up to 1,000 min. For our purposes it may be assumed to decrease slightly over the range of temperatures normally considered.

The constant $B$, on the other hand, is extremely sensitive to temperature, and values increase by a factor of $10^{4}$ or more if one raises the temperature from room temperature to $150{ }^{\circ} \mathrm{C}$. The same temperature increase also reduces the appearance time by a factor of $10^{4}$ according to eq. (6.1.). When the temperature is varied, creep studies will yield graphs similar to figure 1 where $A$ is constant and $B$ is the parameter. 
Figure 6 is a plot of creep data obtained by Derham and co-workers [3] who evaluated $B$ at $50,60,70$ and $80^{\circ} \mathrm{C}$ for three different rubber vulcanizates $1 \mathrm{~mm}$ thick. From the slopes nearly the same values of activation energy-about $(117 \mathrm{kj} / \mathrm{mol})(28 \mathrm{kcal} / \mathrm{mol})$ were obtained for Vulcanizates $\mathrm{G}$ and $\mathrm{H}$ typical accelerated sulfur valcanizates, identical except that Vulcanizate $H$ contained an antioxidant. The value of $B$ for Vulcanizate $G$ at any temperature is about 2.6 times that for Vulcanizate H. Vulcanizate $J$ with an "efficiently vulcanized" system shows a lower activation energy of about $(88 \mathrm{kj} / \mathrm{mol})(21 \mathrm{kcal} / \mathrm{mol})$.

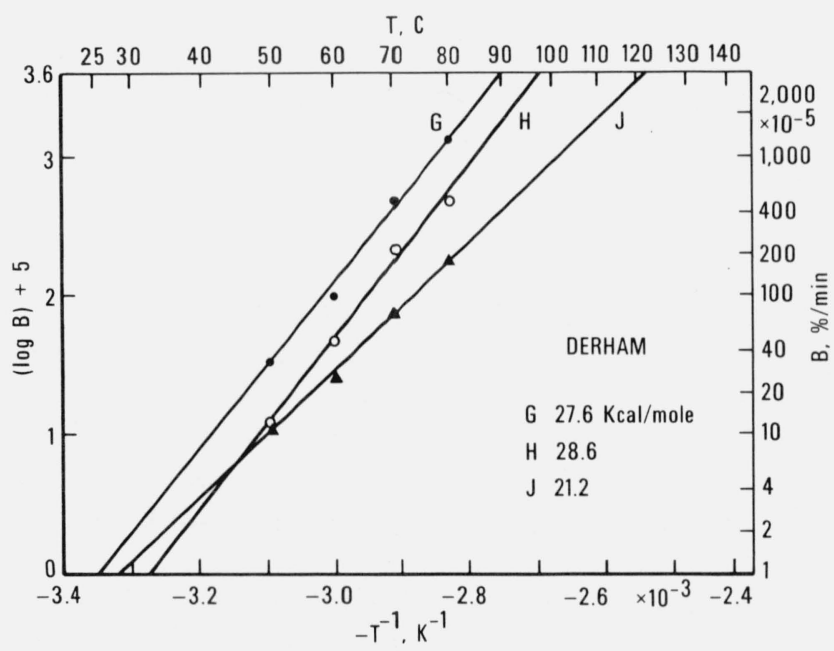

FIGURE 6. $B$ as a function of $T^{-1}$

Data of Derham et al [3]

Equation for Vulcanizate $C$

$5+\log B=-6040\left(T^{-1}-3.348 \times 10^{-3}\right)$

Eq $(9.1)$

The straight line corresponding to Vulcanizate $G$ is represented by the equation

$$
5+\log B=-6040\left(T^{L^{-1}}-3.348 \times 10^{-3}\right)
$$

where $T$ is the temperature in kelvins.

As already mentioned, stress relaxation observations on a natural rubber vulcanizate at temperatures of 100 to $130{ }^{\circ} \mathrm{C}$ made many years ago by Tobolsky, Prettyman and Dillon [20] were reported to yield an activation energy of about $(125 \mathrm{kj} / \mathrm{mol})(30 \mathrm{kcal} / \mathrm{mol})$, corresponding to oxidative chain scission. This may be interpreted to mean that the fundamental mechanism of stress relaxation at 100 to $130{ }^{\circ} \mathrm{C}$ is the same as that for creep at $50-80{ }^{\circ} \mathrm{C}$. A value of $(134$ $\mathrm{kj} / \mathrm{mol})(32 \mathrm{kcal} / \mathrm{mol})$ was obtained from stress relaxation studies at $115-140^{\circ} \mathrm{C}$ by other observers. [30]

The variation of appearance time with temperature for typical Vulcanizate $G$ may be obtained by combining eqs. (6.1) and (9.1) to eliminate $B$. Thus we obtain

$$
\log \left(t_{a}-1\right)=5+6040\left(T^{-1}-3.348 \times 10^{-3}\right)
$$

Figure 7 shows an Arrhenius-type plot of this equation. Of course the slope corresponds to the same activation energy as that given in figure 6 for eq. (9.1).

Values of $t_{a}$ one-tenth of those just given are obtained by the use of eq. (6.2) instead of eq. (6.1).

$$
\log \left(t_{a}-1\right)=4+6040\left(T^{-1}-3.384 \times 10^{-3}\right)
$$

Watanabe [31] after studies which included both creep and stress relaxation, formulated an easily remembered rule that most compounds of natural rubber ( $2 \mathrm{~mm}$ thick) first begin to deviate from linearity with $\log t$ after about 100 hours at $50{ }^{\circ} \mathrm{C}, 10 \mathrm{~h}$ at $70^{\circ} \mathrm{C}$ and $1 \mathrm{~h}$ at $100^{\circ} \mathrm{C}$. These results are plotted as points in figure 7 .

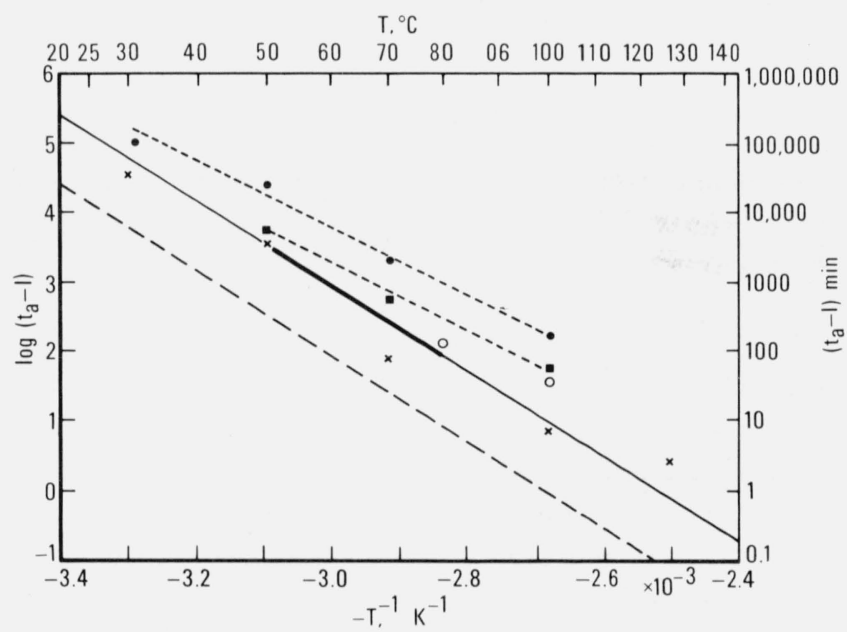

FiguRe 7. Appearance time as a function of temperature

$X$ Aben [16] figure 1, typical natural rubber

- Aben [16] figure 3, ethylene-propylene rubber O Steiner and Tobolsky [33]

$\square$ Watanabe [31]

Lines represent equations derived from data for Vulcanizate $G$ of Derham et. al [3]. Heavy line shows range over which Derham's observations extended

$\log \left(t_{\mathrm{a}}-1\right)=5+6040\left(T^{-1}-3.348 \times 10^{-3}\right)$

Eq. (9.2) obtained from eq. (6.1)

$\cdots \log \left(t_{a}-1\right)=4+6040\left(T^{-1}-3.348 \times 10^{-3}\right)$

Eq. (9.3) obtained from eq. (6.2)

Aben [16], [17] has presented extensive stress relaxation data, which yield interesting correlations of time and activation energies with those obtained from creep data. The slope of the plot of $\Delta F / F_{1}$ against log time is essentially constant up to an "incubation time" or a "time for chemical relaxation to become noticeable" and like the constant $A$ of creep studies this slope is nearly independent of temperature. The slope of the straight line obtained when $\Delta F / F_{1}$ is plotted against $t$ at long times is essentially constant and is highly dependent on temperature. A plot of the logarithm of the "incubation time" against $T^{-1}$ yielded an activation energy of $(109 \mathrm{~kJ} / \mathrm{mol})(26 \mathrm{kcal} / \mathrm{mol})$, nearly the same as Derham's values shown in figure 6. Aben found that values 
of "incubation time" and activation energy were nearly the same for natural rubber as for ethylene-propylene rubber, when each was cross-linked by dicumyl peroxide. Aben found that the times were increased by a factor of several hundred upon the addition of $0.2 \mathrm{phr}$ (parts per hundred of rubber) of antioxidant to the vulcanizate of ethylenepropylene rubber but the slope remained the same. This result is qualitatively similar to Derham's observations with Vulcanizates $G$ and $H$.

Thirion and Chasset [22] measured a quantity which they defined as "the time after which aging prevails." The values obtained for stress relaxation of natural rubber cross-linked by dicumyl peroxide (where Young's Modulus was given as $13.2 \mathrm{~kg} / \mathrm{cm}^{2}$ ) were given as $4800 \mathrm{~min}$ at $76^{\circ} \mathrm{C}$, $1800 \mathrm{~min}$ at $88.3^{\circ} \mathrm{C}$ and $960 \mathrm{~min}$ at $100^{\circ} \mathrm{C}$. These appear to be of the order of 100 times the values of $t_{a}$ at the corresponding temperatures as calculated for Derham's Vulcanizate $G$ by eq. (9.2). Thus they probably should not be considered as values of $t_{a}$. However, the change of these values with temperature over the range 65 to $100{ }^{\circ} \mathrm{C}$ corresponds to an activation energy of $(105 \mathrm{kj} / \mathrm{mol})(25 \mathrm{kcal} / \mathrm{mol})$, nearly the same as that found in Derham's work.

It has long been recognized that at short times physical relaxation effects (including creep) predominate at low (normal ambient) temperatures while chemical effects (including degradation of the network) predominate at high temperatures. The present work on the other hand analyzes the behavior at normal ambient temperatures in terms of creep at short times and creep at long times respectively. It can now be seen that these are merely different aspects of the same general picture. The present analysis predicts that the rapid increase in $B$ with temperature increase calls for a corresponding reduction in appearance time. Phenomena which are long-time at room temperature become short-time phenomena at high temperatures. In the latter case, sometimes one may even fail to note any evidence of the appearance time and linearity limit since they are passed through at very short times before the observations are well underway. Thus there may be no evidence of physical creep, with only chemical creep apparent throughout the whole period of observation. However there is no reason to doubt that both physical and chemical creep are occurring at all temperatures and all times.

A temperature of $65^{\circ}$ or $70^{\circ} \mathrm{C}$ has sometimes been set [22, 32,33 ] as that below which physical effects (e.g. viscoelasticity) predominate in natural rubber vulcanizates and above which chemical effects (e.g. degradation) are more important. In the present context as shown in figure 7 this merely means that, depending on the sensitivity of the measurement, an appearance time of the order of 20-200 min has been chosen to separate the two regions of temperature. Attention is then being devoted chiefly to the behavior at longer times.
The discussion up to this point has assumed that the quantity $B$ is determined by a single reaction with a constant activation energy at all temperatures. If this is not the case for certain systems, straight lines of constant slope will not be obtained in plots like figure 4 showing the chemical creep as a function of time. Likewise values of $B$ obtained at different temperatures will not yield straight lines like those in figure 6. Gent, [2] as already mentioned, observed a constantly increasing slope at $60^{\circ} \mathrm{C}$ for natural rubber crosslinked by dicumyl peroxide and ascribed it to auto-catalysis [27]. The data of Wood and Bullman [9], replotted for the present study as already mentioned in Section 6, showed constant slopes for the same system at $24^{\circ} \mathrm{C}$. In another instance as already mentioned in Section 8, Gent [2] found that $B$ increased with time before coming constant. $\mathrm{He}$ ascribed this behavior to a change in structure of the rubber during the experiment.

In view of the parallelism between creep and stress relaxation discussed in section 7 it may be concluded that $B$ will not be found constant for several systems where stress relaxation has indicated changes in mechanism with a resulting change in activation energy with temperature [16, 17]. Although attempts have been made to separate the stress relaxation effects arising from the different mechanisms apparently no analogous attempts have been made with the creep data.

The general observation that mechanical behavior at long times can be duplicated or approximated by similar behavior at higher temperature is well known. It is, of course, the basis for many investigations of time-temperature equivalence with shifts to yield master curves of various properties. In constrast with the present work most of these studies have dealt with reversible physical effects denoted as viscoelastic and considered to be related to the approach to the glass transition as the temperature is reduced.

One study of this sort at the NBS [13] involved indentation measurements of compliance as a function of time and temperature at temperatures from -67 to $+100{ }^{\circ} \mathrm{C}$. It yielded a relationship between equivalent time and temperature which corresponds to an activation energy of (154.5 $\mathrm{kJ} / \mathrm{mol})(36.9 \mathrm{kcal} / \mathrm{mol})$. This value did not vary with extent of cross-linking. Reference should be made to this earlier paper [13] for a discussion and a comparison with other published values of activation energy including those predicted by the Williams, Lamdel and Ferry equation. For the most part these values of activation energy are all somewhat larger than those noted here.

The present results, dealing with chemical degradation at higher temperatures and longer times, would not appear to be explainable in terms of viscoelastic effects related to the glass transition. Furthermore, the difference in calculated activation energies would indicate that different processes are involved. However, both viscoelastic and degradative 
mechanisms apparently lead to the conclusion that the main effect of an increase in temperature may be simply a decrease in the time scale. In both cases cross-linking does not seem to play a major role.

\section{ASTM test for aging}

ASTM Method D1206-52T [28] was a method of test (proposed some years ago as a tentative method and discontinued in 1964) to determine resistance to aging of rubber vulcanizates. Measurements were to be made of the difference between the observed creep at elevated test temperatures $\left(100,120\right.$ and $\left.140^{\circ} \mathrm{C}\right)$ and the extrapolated value of the initial creep proportional to $\log t$. The latter quantity was determined by drawing a tangent at the 1-minute value. This difference at some arbitrary time was to be used as a measure of the relative resistance to aging of different rubbers.

The method was discontinued when it was found that in several instances most of the secondary creep was recoverable upon release of the load. This was in agreement with observations of Gent [2] and Roth, Bullman, and Wood [13]. Thus it was felt that it could not be used as a measure of degradation of the network during aging. Likewise during normal aging the specimen is maintained in the unstretched condition.

\section{Pre-rupture Behavior}

At high stresses near the breaking point there have been reports of creep in excess of that predicted by eq. (1.1). Lyons [1] mentions this as characteristic of a loosening of structure observed with a number of different materials. Braun [34], working with stretched rubber bands, found regions of fractional creep which were first linear with $\log t$, then linear with $t$, and finally a region of more rapid rise beginning at 120-240 days and culminating in rupture at about 240 days.

Figures 8 and 9 present for comparison with Figures 3 and 4 the results of experiments performed in our own laboratories. The conditions of the experiment were described in our previous paper [6], but the results were not given graphical representation in that publication. The specimen (called Specimen C in the previous work) was the only one in which observations were continued until the specimen broke. This occurred after about 60 days $(90,000 \mathrm{~min}$.) of creep. The value of $B$ found here $\left(125.1 \times 10^{-5} \% / \mathrm{min}\right.$.) was the largest reported in the previous paper, while the value of $A(2.874 \%$ /unit $\log t)$ was not very different from that for the other specimens. It is clear that, for an appreciable time

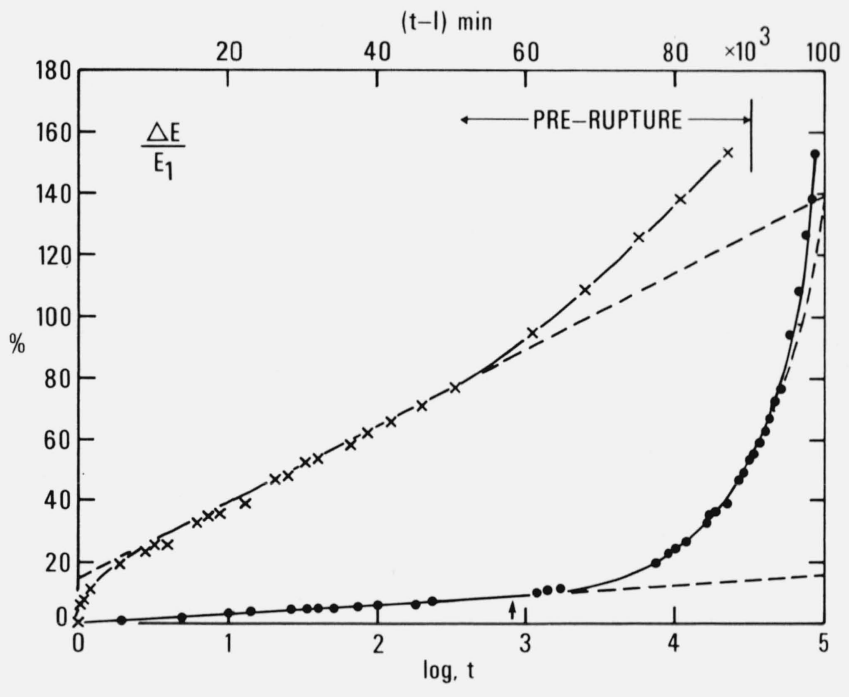

FIGURE 8. Fractional creep as a function of $\log \mathrm{t}$ or of $(\mathrm{t}-1)$ showing prerupture behavior

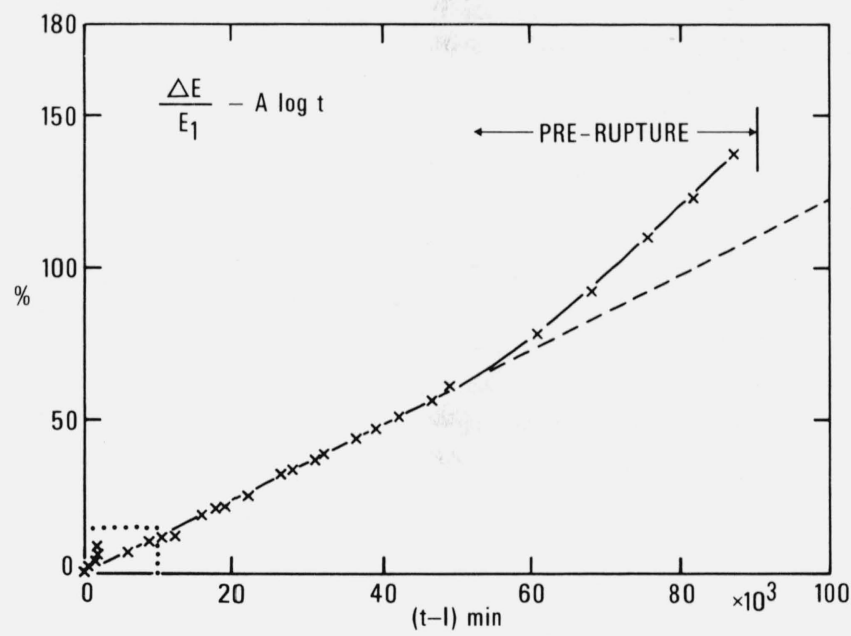

FIGURE 9. Chemical creep showing pre-rupture behavior $\left(\Delta E / E_{1}-A \log t\right)$ as a function of $(t-1)$
$A=2.874$ percent/unit $\log t$ $A=2.874$ percent/unit $\log t$

before the specimen broke, the creep exceeded that predicted by eq (1.1) with constants $A$ and $B$ determined from the data at shorter times. The amount of the excess can be determined most readily as the deviation from the dashed line in figure 9. The excess can also be determined as the deviation from the dashed line in figure 8 , but the line there is not as unambiguously determined as the line in figure 9.

Further work on pre-rupture creep would be highly desirable. Research in this field might be expected to lead to the development of a method for predicting rupture well in advance of its occurrence. 


\section{Conclusions}

Creep may usually be represented by the equation

$$
\Delta E / E_{1}=A \log t+B(t-1)
$$

where $\Delta E$ is the increase of elongation above $E_{1}$, the oneminute value, during the time $t$.

It is convenient to show experimental values of $\Delta E / E_{1}$ on plots with one abscissa $\log t$ and a second abscissa $(t-1)$. The initial slope of the first plot is $A$, a measure of physical creep. The limit of the final slope of the second plot is $B$, a measure of chemical creep. After $A$ has been determined from the first plot a plot can be made showing $\left(\Delta E / E_{1}-\right.$ $A \log t)$ as a function of $(t-1)$. If the equation is valid the plot will be linear with a slope equal to $B$. This is found to be the case in almost every instance, and the slope can be determined from only the early observations if desired. When the equation is valid an alternative procedure can be used to determine the two constants directly from the data by solving simultaneous equations. The minimum number of observations required by this method is only three. Prerupture creep in excess of that predicted by the equation is sometimes observed.

The constant $A$ is usually between 1 and 10 percent/unit $\log t$. It is strongly dependent on cross-linking but nearly independent of temperature. On the other hand, $B$, is nearly independent of cross-linking but srongly dependent on temperature. It increases by a factor of the order of $10^{4}$ as the temperature is raised from 25 to $150{ }^{\circ} \mathrm{C}$. This change corresponds to an activation energy of $(85-125 \mathrm{kj} / \mathrm{mol}) 20$ - $30 \mathrm{kcal} / \mathrm{mol}$ ). From this one concludes that it probably reflects oxidative degradation of the network.

The exact value of $B$ is highly dependent on the thickness of the specimen and may even approach zero for a specimen of infinite thickness. It is highly sensitive to the amount of ozone in the ambient air. Values of $B$ in the literature range from about $0.1 \times 10^{-5}$ to $10,000 \times 10^{-5} \mathrm{percent} / \mathrm{min}$. The lowest value is equivalent to less than 1 per cent per year.

An appearance time $t_{a}$ defined so that at $\left(t_{a}-1\right) \Delta E / E_{1}$ exceeds $A \log t$ by unity, should make $\left(t_{a}-1\right)$ equal to $B^{-1}$. Available literature data confirm this reciprocal relationship reasonably well. Increases in temperature, affecting $B$ and not $A$, thus give rise to drastic reductions in appearance time. Thus phenomena which occur at long times at $25^{\circ} \mathrm{C}$ appear as short-time phenomena at high temperatures. However, for many typical systems both chemical and physical creep normally appear to continue without change of rate at all temperatures at which observations have been reported and at all reported times up to periods of several years.

\section{References}

[1] Lyons, W. J. The general relations for flow in solids and their applications to the plastic behavior of tire cords, J. Applied Phys. 17, 472 (1946).

[2] Gent, A. N., Relaxation processes in vulcanized rubber, II. Secondary relaxation due to network breakdown, J. Applied Polymer Sci. 6, 442 (1962). Rubber Chem. Technol. 36, 389 (1963).

[3] Derham, C. J., Southern E., and Thomas, A. G., Some factors affecting the life of rubber articles in service, paper presented at International Rubber Conference, Moscow, Nov. 1969; NRPRA Rubber Technol. Rubber Develop, Supplement No. 7, 1 (1970).

[4] Derham, C. J., Creep and stress relaxation and their relevance to engineering applications. NRPRA Third Rubber in Engineering Conference 1973 page F/1.

[5] Derham, C. J., Creep and stress relaxation of rubbersthe effects of stress history and temperature changes, J. Materials Sci., 8, 1023 (1973).

[6] Wood, L. A., Bullman, G. W., and Roth, F. L., Long-time creep in a pure-gum rubber vulcanizate: influence of humidity and atmospheric oxygen, J. Res. Nat. Bur. Stand. (U.S.), 78A, No. 5, (Phys. and Chem.), 623-629 (Sept.-0ct. 1974). Rubber Chem. Technol. 48, 134 (1975).

[7] Gent, A. N., Relaxation processes in vulcanized rubber, I. Relation among stress relaxation creep, recovery, and hysteresis, J. Applied Polymer Sci. 6, 433 (1962). Rubber Chem. Technol. 36, 377 (1963).

[8] Martin, G. M., Roth, F. L., and Stiehler, R. D., Behavior of "pure gum" rubber vulcanizates in tension, Trans. Inst. Rubber Ind. 32, 189 (1956), Rubber Chem. Technol. 30, 876 (1957).

[9] Wood, L. A. and Bullman, G. W., Creep and other tensile properties of rubber cross-linked by dicumyl peroxide, J. Polymer Sci. A-2 10, 43 (1972).

[10] Brenschede, W., Deformation mechanics of rubber-like bodies. Extension of concepts of the kinetics of rubber elasticity, Kolloid-Zeits 104, 1 (1943).

[11] Wildschut, A. J., Technological and Physical Investigations on Natural and Synthetic Rubbers, page 120, (Elsevier Publishing Co., New York, 1946).

[12] Wood, L. A. and Roth, F. L., Creep of pure-gum rubber vulcanizates from indentation-time measurements, Proc. 4th Rubber Technol. Conf. London 1962 p. 328. Institution of Rubber Industry London 1963. Rubber Chem. Technol. 36, 611 (1963).

[13] Roth, F. L., Bullman, G. W., and Wood, L. A., Compliance-time-temperature relationships from indenta- 
tion measurements on a pure-gum rubber volcanizate, J. Res. Nat. Bur. Stand. (U.S.) 69A, No. 4, (Phys. and Chem.) 347-353 (July-Aug. 1965). Rubber Chem. Technol. 39, 397, (1966).

[14] Wood L. A., Creep of pure-gum valcanizates of natural rubber, J. Rubber Res. Inst. Malaya 22, 309 (1969); Rubber Chem. Technol. 43, 1482 (1970).

[15] Metherell, C., Effect of ozone on creep of vulcanizates, J. Polymer Sci. Polymer Phys. Ed. 16, 813 (1978).

[16] Aben, W. J. G. M., Prediction of the long-time behavior of deformed rubbers, Rev. Gen. Caoutchouc Plast. 51, 727 (1974) (French).

[17] Aben, W. J. G. M., The long-time behavior of deformed rubbers, Plastica 27 (3) 82 (1974) (Dutch).

[18] Derham, C. J., Creep of dicumyl peroxide crosslinked natural rubber., J. Polymer Sci. Polymer Phys. Ed. 13, 1855 (1975).

[19] Meier, J. F., Rudd, G. E., and Weir, D. F., Creepresistant elastomer formulations, Rubber Chem. Technol. 52, 50 (1979).

[20] Tobolsky, A. V., Prettyman, I. B., and Dillon, J. H., Stress relaxation of natural and synthetic rubber stocks, J. Applied Phys. 15, 380 (1944) Rubber Chem. Technol. 17, 551 (1944).

[21] Tobolsky, A. V., Properties and Structure of Polymers, page 223, (John Wiley \& Sons, New York, 1960).

[22] Thirion, P., and Chasset, R. Relative contributions of viscoelasticity and ageing to the relaxation of rubber vulcanizates, Proc. 4th Rubber Tech. Conf. London 1962 T. H. Messenger, Ed. page 338 (Inst. Rubber Ind. London 1963) Rev. Gen. Caoutchouc 41, 271 (1964); Rubber Chem. Technol. 36, 50 (1963).

[23] Chasset, R., and Thirion, P., Viscoelastic relaxation of rubber vulcanizates between the glass transition and equilibrium. Proc. Int. Conf. Non-crystalline Solids Delft 1964; J. A. Prins Ed. page 345. NorthHolland Publishing Co., Amsterdam. Interscience Publishers, New York; Rubber Chem. Technol. 39, 870 (1966), Rev. Gen Caoutchouc 44, 1041 (1967).

[24] Chasset, R. and Thirion, P., Delayed elasticity of pure- gum rubber vulcanizates, J. Rubber Res. Inst. Malaya 22, 331 (1969).

[25] Tamura, S. and Murakami, K., Chemorheology of irradiation-cured natural rubbers: 1 . Stress relaxation mechanisms for various curing systems in natural rubber at high temperatures, Polymer 14, 569 (1973). Rubber Chem. Technol. 48, 141 (1975).

[26] Murakami, K., Recent chemorheological studies on rubber vulcanizates, Rubber Chem. Technol. 48, 913 (1975).

[27] Dunn, J. R., Scanlan, J., and Watson, W. F., Stress relaxation during the thermal oxidation of vulcanized natural rubber. Trans. Faraday Soc. (436) 55, 667 (1959) Rubber Chem. Technol. 33, 423 (1960).

[28] American Society for Testing and Materials, Tentative Method of Test for Resistance to Aging of Vulcanized Rubber by Measurement of Creep, ASTM Designation D 1206-52T.

[29] Wood, L. A., Bullman, G. W., and Decker, G. E., Modulus of natural rubber cross-linked by dicumyl peroxide. I. Experimental observations, J. Res. Nat. Bur. Stand. (U.S.) 76A, No. 1 (Phys. and Chem.), 51-59 (Jan.-Feb. 1972). Rubber Chem Technol. 45, 113 (1972).

[30] Berry, J. P., and Watson, W. F., Stress relaxation of peroxide and sulfur vulcanizates of natural rubber. J. Polymer Sci. 18, 201 (1955).

[31] Watanabe, K., Stress relaxation and creep of several vulcanized elastomers, J. Soc. Rubber Ind. Japan 33, 882 (1960 (Japanese), Rubber Chem. Technol. 35, 182 (1962).

[32] Wood, L. A., and Roth, F. L., Stress-temperature relations in a pure-gum vulcanizate of natural rubber, J. Applied Phys. 15, 781 (1944), Rubber Chem. Technol. 18, 367 (1945).

[33] Steiner, G., and Tobolsky, A. V., A note on stress relaxation of rubber networks in the vicinity of room temperature, Rubber Chem. Technol. 43, 1036 (1970).

[34] Braun, M. L., A preliminary study of the after-effect or drift in rubber under constant load, Physics 7, 421 (1936), Rubber Chem. Technol. 10, 224.(1937). 
